\title{
Immune Checkpoint Inhibitors in Metastatic Colorectal Cancer: Current Status, Recent Advances, and Future Directions
}

\author{
Saivaishnavi Kamatham ${ }^{1} \cdot$ Faisal Shahjehan $^{2} \cdot$ Pashtoon M. Kasi $^{3}$
}

Published online: 10 July 2019

(C) The Author(s) 2019

\begin{abstract}
Purpose of Review Immune checkpoint inhibitors have shown very promising outcomes in the subset of metastatic colorectal cancers (CRCs) that are mismatch repair deficient (dMMR)/microsatellite instability-high (MSI-H). We reviewed the existing literature on immune checkpoint inhibitors in colorectal cancers to highlight the recent advances and future directions.

Recent Findings The significance of immune check point inhibitors in dMMR/MSI-H metastatic CRCs has been validated by several studies employing anti-programmed death cell receptor (anti-PD1) and anti-PDL1 antibodies single agent or in combination with anti-CTLA4 antibodies ("KEYNOTE" and "CHECKMATE" studies). This has led to FDA approval of these drugs. At present, its approval is limited to those who have failed traditional chemotherapy (5-FU, oxaliplatin, and irinotecan). The trials for the dMMR/MSI-H CRCs are now being moved up in terms of first-line, neoadjuvant, and adjuvant settings.

Summary The results of various studies favor the excellent antitumor activity and safety profile of anti PD-1 monoclonal antibodies that include pembrolizumab and nivolumab in MSI-H colorectal cancer. Combination immunotherapy with nivolumab and ipilimumab has also shown significant clinical benefit in dMMR/MSI-H metastatic CRC patients. These have now been FDA-approved. Multiple ongoing studies assessing the safety and efficacy of other anti-PD/PD-L1 agents (e.g., durvalumab, atezolizumab) alone or in combination therapy with classes of drugs are in progress. The goal ideally would be to identify approaches to expand activity of immune checkpoint inhibitors beyond the dMMR/MSI-H subset of CRC, which represent $4-5 \%$ of metastatic CRC patients. These are also being moved up in terms of usage in earlier lines of therapy and neoadjuvant/adjuvant approaches.
\end{abstract}

Keywords Immunotherapy $\cdot$ Immune checkpoint inhibitors $\cdot$ Metastatic colorectal cancer $\cdot$ Microsatellite instability $\cdot$ Mismatch repair deficiency · Keynote studies · Checkmate studies · Pembrolizumab · Nivolumab - Ipilimumab · Durvalumab . Atezolizumab $\cdot$ Tremelimumab $\cdot$ Avelumab $\cdot$ Cancer vaccines

This article is part of the Topical Collection on Systemic Therapies in Colorectal Cancer

Pashtoon M. Kasi

pashtoon-kasi@uiowa.edu

Saivaishnavi Kamatham

kamatham.vaishnavi@mayo.edu

Faisal Shahjehan

dr.fsj1@gmail.com

1 Visiting Research Fellow, Division of Hematology/Oncology, Mayo Clinic, 4500 San Pablo Rd S, Jacksonville, FL 32224, USA

2 CORPS Program, Division of Hematology/Oncology, College of Medicine and Oncology, Gastrointestinal Malignancies, Mayo Clinic, 4500 San Pablo Rd S, Jacksonville, FL 32224, USA

3 College of Medicine and Oncology, University of Iowa, 200 Hawkins, Iowa Cit, IA 52242, USA

\section{Introduction}

Colorectal cancer (CRC) is the third leading type of cancer in the USA in both males and females [1]. The major treatment modalities for CRC have been surgery, chemotherapy, and radiation therapy. The overall survival (OS) of patients with metastatic CRC has significantly improved over the last few decades. It has not been just one drug or class of drugs. The progress has been due to multiple drugs, chemotherapies, biologics, targeted therapies, and now immunotherapy, alone or in combination that have led to the improvement in OS with metastatic CRCs. This has been paralleled with advancements in surgical, radiation, and interventional oncology disciplines.

Of particular note is the activity and approval of immunotherapy for a subset of colorectal cancers that are mismatch repair deficient (dMMR) or microsatellite 
instability high (MSI-H) tumors. The dMMR/MSI-H could be a germline event (Lynch syndrome) or somatic (tumor-Lynch). These are exquisitely responsive to immune checkpoint inhibitors due to the "hyper-mutated" nature of these tumors. The dMMR/MSI-H tumors on average have $>1700$ mutations as opposed to approximately 70 in sporadic CRCs.

Overall, depending on the studies/cohorts reported, around 10-20\% have DNA mismatch repair deficiency (dMMR/MSI-H tumors) [2, 3]. In the metastatic setting, these represent about $4-5 \%$ of cases. What is unique about this subset of tumors is that have a poor response to chemotherapy [4]. However, the same tumor biology makes it inherently resistant to chemotherapy; because of their hypermutated nature, these tumors benefit immensely to immunotherapy with immune checkpoint inhibitors, e.g., pembrolizumab, nivolumab, and ipilimumab [5-8]. In this article, we aim to review the role of immune checkpoint inhibitors in advanced metastatic CRC patients, recent advances, and future directions.

\section{Testing for Mismatch Repair Deficiency/Microsatellite Instability in CRC}

The subset of patients with mismatch repair deficiency can be identified through various testing platforms/methodologies. Most of the national and international consensus guidelines and institutions do immunohistochemistry (IHC) for mismatch repair deficiency. Where there is discrepancy or high suspicion, microsatellite instability (MSI) testing can also be done (PCR-based assay) [9]. The hypermutated nature of these tumors can now also be noted on commercially available next generation sequencing (NGS) testing platforms that report the high tumor mutation burden (TMB-High). For stage-II patients, identification of dMMR/MSI-H identifies the cohort that would not benefit from chemotherapy as well as those who may have underlying Lynch syndrome [10]. The immune checkpoint inhibitors at present are approved for the metastatic dMMR/MSI-H subset of CRC patients [6]. Of note, while any of the three commonly used testing strategies, i.e., dMMR through IHC or MSI$\mathrm{H}$ through PCR or now TMB through NGS-based assays identify this subset of CRC patients, it is not a perfect overlap. There are small subsets who are microsatellite stable (MSS) and mismatch repair proficient (pMMR) who may still be hypermutated and be TMB-High (e.g., patients with POLE mutations). This is an area of ongoing research with trials specifically seeking patients who have these tumor characteristics. Theoretically, they should respond similar to dMMR/MSI-H patients.

\section{Role of Immune Checkpoint Inhibitors in MSI-H Metastatic CRC}

Immune checkpoint inhibitors target the checkpoint molecules such as PD1, PD-L1, or CTLA-4, thereby leading to the immunotherapy response to the cancer cells [8]. Other targets and combination of these agents with other agents are being explored. Following is an account of the data thus far on the use of anti-PD1 alone or in combination with anti-CTLA-4 agents.

\section{Pembrolizumab Monotherapy}

The efficacy of pembrolizumab in patients with metastatic CRC has been well studied by Le et al. A phase 2 multicenter clinical trial was conducted with 41 patients who were divided into three cohorts: (i) patients with dMMR CRC, (ii) patients with pMMR CRC, and (iii) patients with dMMR cancer other than CRC. Ten milligrams per kilogram of pembrolizumab was administered intravenously every 2 weeks. They reported a $40 \%$ immune-related objective response rate (ORR) and the 20-week immune-related progression-free survival rate (PFSR) was noted to be $78 \%$ for dMMR CRC. In sharp contrast, the ORR and PFSR for pMMR CRC patients was found to be 0 and $11 \%$ respectively, thereby emphasizing the clinical activity of pembrolizumab specifically for the dMMR CRC but not the for pMMR CRC. The authors in the same study also reported the somatic mutation load in dMMR versus pMMR CRCs. This was estimated to be a mean of $1782 \mathrm{mu}-$ tations per tumor in dMMR as opposed to only 73 mutations per tumor in pMMR CRCs. The high mutation load was associated with prolonged PFS [11].

KEYNOTE-028 trial, which is an international, multi cohort, multicenter, non-randomized, open label phase Ib study assessed the anti-tumor activity and safety profile of pembrolizumab in 20 PD-L1 positive metastatic solid tumors. Thirty-three (24\%) of the 137 patients with CRC were PDL-1 positive of which only 23 patients were enrolled in the study. After a median follow-up of 5.3 months, the results showed that pembrolizumab had a reasonable safety profile with treatment-related adverse events of fatigue, asthenia, and stomatitis in $35 \%$ of the patients with no grade 3 adverse events noted. However, the antitumor activity was seen in only one patient and not surprisingly, the tumor was noted to be dMMR/MSI-H [12].

KEYNOTE-164 study documented reported the antitumor activity of pembrolizumab in 61 patients with dMMR/MSI-H colorectal cancer. Two hundred milligrams of pembrolizumab was administered once every 3 weeks. The results after a median follow-up of 7.4 months demonstrated an impressive objective response rate of $42.9 \%$ and a disease control rate of $50.8 \%[13 \bullet \cdot]$. What is very encouraging and a recurring theme 
is not only the initial response but also ongoing response and durability of responses in these studies.

\section{Nivolumab Monotherapy}

Similar to studies on pembrolizumab, interim results of the CheckMate-142 (NCT02060188) study also revealed promising results with anti-PD1 therapy with nivolumab monotherapy as well in dMMR/MSI-H metastatic CRCs [14]. In the 33 MSI-H patients who received nivolumab $3 \mathrm{mg} / \mathrm{kg}$ every 2 weeks, $82 \%$ had $\geq 2$ prior regimens and $15 \%$ of them were $B R A F-V 600 E$ positive. Investigator reported ORR was of $27 \%(n=9)$. None of the patients had complete responses, with $27 \%(n=9)$ them having confirmed partial responses. The interim results showed a median progression free survival (PFS) of 5.3 months, 4-month PFS rate of 55\%, median overall survival (OS) of 16.3 months, 5-month OS rate of $75 \%$. Seventy-nine percent $(n=26)$ of the patients had treatmentrelated adverse events (TRAEs) of which diarrhea (27\%) and fatigue $(27 \%)$ were most common. Only 7 patients had grades 3-4 TRAEs, as summarized in Table 1.

\section{Combination Immunotherapy (Nivolumab plus Ipilimumab)}

The clinical efficacy of combination immunotherapy with nivolumab plus ipilimumab, which is an anti-CTLA-4 monoclonal antibody, has been presented in the CheckMate-142/ NCT02060188 - a large, multicenter, open-label phase II trial involving $\mathrm{mCRC}$ patients with $\mathrm{dMMR} / \mathrm{MSI}-\mathrm{H}$ patients from eight countries. The patients in the study $(n=119)$ received nivolumab $3 \mathrm{mg} / \mathrm{kg}$ IV infusion and ipilimumab $1 \mathrm{mg} / \mathrm{kg} \mathrm{IV}$ infusion once every 3 weeks for four doses followed by nivolumab $3 \mathrm{mg} / \mathrm{kg}$ IV once every 2 weeks unless they had disease progression, severe adverse events, death, or end of study and were followed for median duration of 13.4 months. The outcomes of the study showed an ORR of $54.6 \%(95 \%$ CI, 45.2 to 63.8 ) according to the investigator, ORR of $49 \%$ (95\% CI, 39.5 to 58.1) according to blinded independent central review and disease control rate (DCR) of $80 \%(95 \% \mathrm{CI}$, 71.5 to 86.6). In addition, the 9 and 12 month progression-free survival rates (PFS) were $76 \%$ and $71 \%$ respectively and the 9 and 12 month overall survival rates were $87 \%$ and $85 \%$ respectively. Thirty-two percent of patients had grades 3-4 manageable adverse events. Combination immunotherapy, thus results in improved efficacy compared with anti-PD-1 monotherapy for dMMR/MSI-H mCRC [15••]. Side effects as noted are higher as opposed to single-agent anti-PD1 therapy. However, these are manageable. Also, it is important to note that there are different dosing regimens employing antiCTLA4 alongside anti-PD1 therapy. Low-dose ipilimumab
("Ipi-light" or "Ipi-1") employing the $1 \mathrm{mg} / \mathrm{kg}$ dosing is lower than the dosing used in the initial studies employing this agent. Learning from clinical trials and our colleagues in melanoma and lung cancer, the lower dosing's adverse events are manageable.

At present, however, with excellent responses to immunotherapy with single-agent anti-PD1, combination immunotherapy even though FDA-approved is not readily used unless dealing with situations where response is the key, e.g., symptomatic/visceral crises cases. Anecdotal cases of patients who have failed single agent anti-PD1 have responded later to combination immunotherapy.

The late-breaking results from the CheckMate-142 study/LBA18_PR were presented at the European Society for Medical Oncology (ESMO) in October 2018. Forty-five patients with no prior therapy for $\mathrm{dMMR} / \mathrm{MSI}-\mathrm{H} \mathrm{mCRC}$ were given the combination immunotherapy with nivolumab $3 \mathrm{mg} / \mathrm{kg}$ every 2 weeks and a low dose of ipilimumab $1 \mathrm{mg} / \mathrm{kg}$ every 6 weeks. After a median follow-up of 13.8 months, the overall response rate (ORR) was $60 \%$, disease control rate (DCR) was $84 \%$, and a complete response rate was $7 \%$. Twelve-month progression-free survival rates (PFS) and overall survival (OS) rates were $77 \%$ and $83 \%$ respectively. Sixteen percent of the patients had grades 3-4 treatment-related adverse events. The study results established that nivolumab every 2 weeks with a low dose of ipilimumab every 6 weeks demonstrated a strong, durable, and well-tolerated clinical value as a first-line therapy for $\mathrm{dMMR} / \mathrm{MSI}-\mathrm{H} \mathrm{mCRC}$ and may epitomize a new first-line treatment choice for this subset of patients $[16 \bullet \bullet$.

LBA37_PR study by Chalabi et al. presented at ESMO 2018 is the first study to elaborate the safety and feasibility of neoadjuvant ipilimumab plus nivolumab therapy in early stage dMMR and MMR proficient (pMMR) colon cancers. Fourteen patients of whom 8 had pMMR and 7 had dMMR early stage colon cancer received a low dose of ipilimumab at $1 \mathrm{mg} / \mathrm{kg}$ on day 1 and nivolumab at $3 \mathrm{mg} / \mathrm{kg}$ on days 1 and 15 . One hundred percent of the dMMR patients showed a robust pathologic response with $<5 \%$ viable tumor cells. Patients with pMMR tumors did not show a major pathological response, but demonstrated a significant rise in CD8+ $\mathrm{T}$ cell infiltration. Therefore, the study recommended that shortterm neoadjuvant immunotherapy with ipilimumab plus nivolumab could be a new strategy for patients with dMMR colon cancer $[17 \bullet \bullet]$.

Immune checkpoint inhibitors are currently approved only for patients with dMMR/MSI-H metastatic colorectal cancer patients, after failure of standard of care chemotherapy with 5fluorouracil (5-FU), oxaliplatin, and irinotecan. Current NCCN guidelines on their administration and dosage are elaborated in Table 2. 


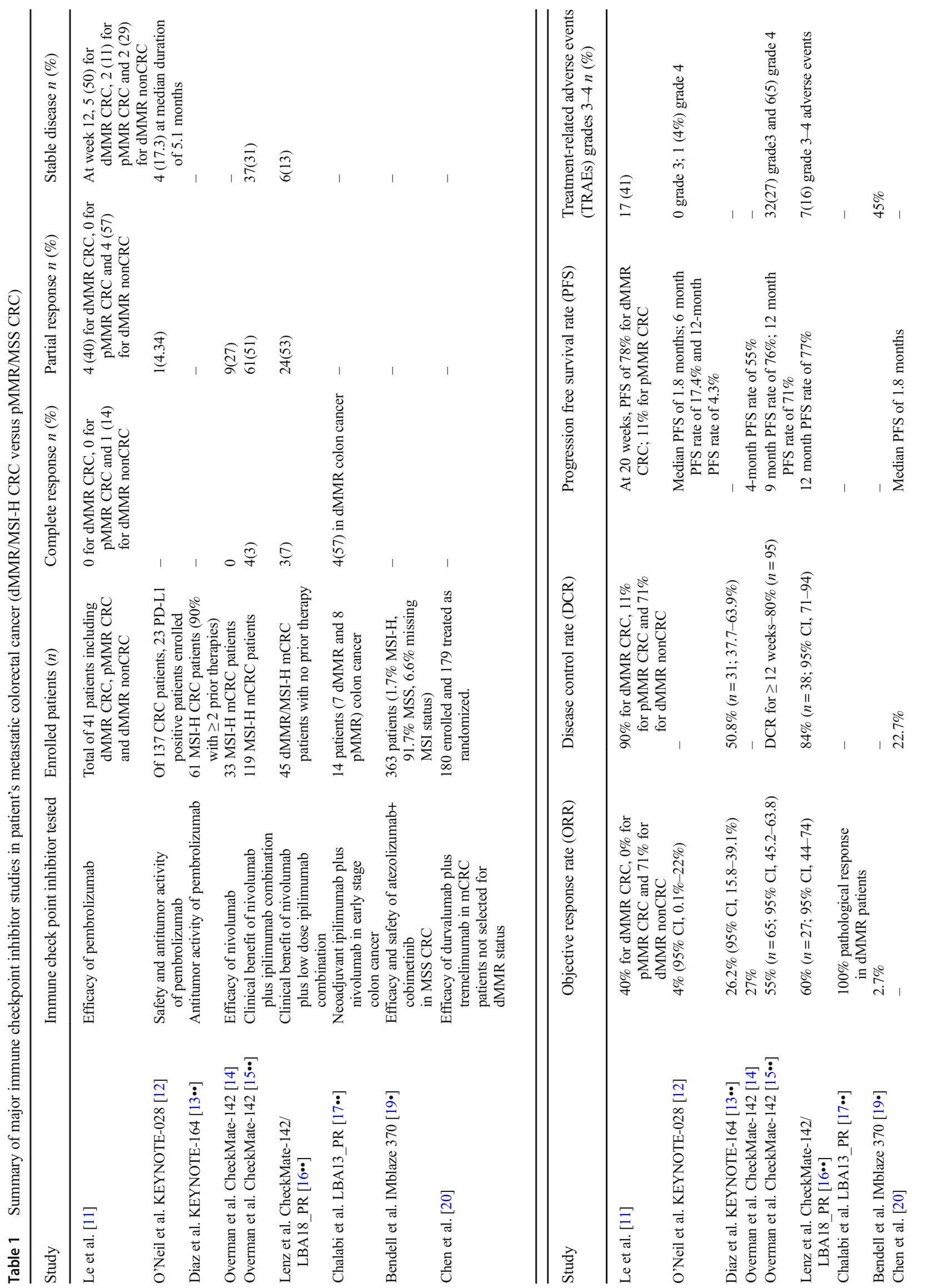


Table 2 Current NCCN guidelines for use of immune check point inhibitors in metastatic CRC. Of note, its use is only approved for patients with $\mathrm{dMMR} / \mathrm{MSI}-\mathrm{H}$, and at present is after failure of chemotherapy with 5-FU, oxaliplatin, and irinotecan [18]*

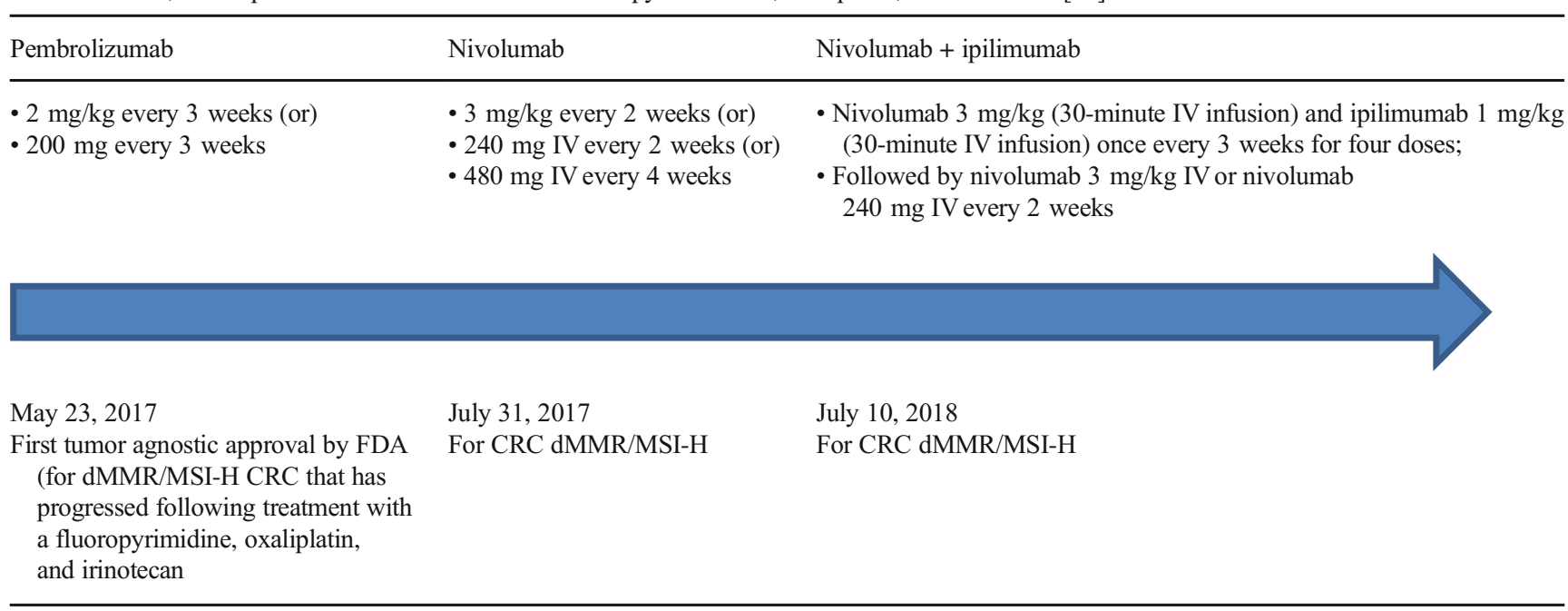

*Of note, it now allows for usage of immunotherapy if someone is not a candidate for chemotherapy

\section{Immune Check Point Inhibitors and MSS Colorectal Cancer}

Atezolizumab is an anti PDL1 monoclonal antibody. IMblaze 370 study by Bendell et al. compared if atezolizumab in combination with cobimetinib, a MEK1/MEK2 inhibitor may favor better anti-tumor activity than atezolizumab monotherapy or the standard of care regorafenib in patients with MSS/MSI-L metastatic colorectal cancer. Three hundred sixty-three patients were enrolled of which $1.7 \%$ were MSI$\mathrm{H}$ mCRC, $91.7 \%$ were MSS, and 6.6\% had missing MSI status. They were randomized in 2:1:1 ratio to receive atezolizumab+cobimetinib, atezolizumab monotherapy, or regorafenib, respectively. Median overall survival was 8.9 months, 7.1 months, and 8.5 months; overall response rates were $2.7 \%, 2.2 \%$, and $2.2 \%$ for atezolizumab+ cobimetinib, atezolizumab, and regorafenib monotherapy respectively. Their results indicated that atezolizumab with cobimetinib did not establish statistically significant improved overall survival, progression-free survival and overall response rate compared with either of the control arms [19•].

A study by Chen et al. presented at ASCO GI symposium 2019 demonstrated the significance of combining durvalumab, a PD-L1 receptor antibody plus tremelimumab, an anti CTLA-4 monoclonal antibody with best supportive care (BSC) versus BSC alone in patients with refractory, metastatic CRC not selected for dMMR status. The study did not include patients with known dMMR status. One hundred seventy-nine patients randomized to the treatment arm were administered durvalumab $1500 \mathrm{mg}$ on day 1 every 28 days and tremelimumab $75 \mathrm{mg}$ on day 1 for the first four cycles. After a median follow-up of 15.2 months, the median overall survival was 6.6 months and 4.1 months for the treatment arm and BSC arm respectively. The disease control rate in the treatment and control arms were $22.7 \%$ and $6.6 \%$ respectively. Moreover, lower deterioration and well-maintained quality of life were seen in the treatment arm, although grades 3-4 adverse events were higher in the treatment arm as summarized in Table 1 [20].

\section{Radiotherapy With Immunotherapy in Colorectal Cancers}

In metastatic cancers, radiotherapy can to be a powerful adjuvant for immunotherapy, amplifying the clinical benefits of immunotherapy to improve survival $[21,22]$. Ongoing clinical trials combining immunotherapy with radiation or other locoregional modalities are summarized in Table 3.

\section{Conclusion/Future Directions}

At present, immune checkpoint inhibitors have shown promising and durable activity for dMMR/MSI-H CRCs. This has led to FDA approval of anti-PD1 agents alone and now more recently in combination with anti-CTLA4 therapy. However, these as noted earlier, only represent 4-5\% of metastatic cancer patients. These agents alone have not shown activity for the pMMR/MSS CRC patients. As noted in some of the studies in Table 3, combination with other classes of immunotherapy, locoregional treatments and/or targeted therapies is being explored. Immunotherapy alongside MEK-inhibition initially was hypothesis generating for pMMR/MSS metastatic CRC 

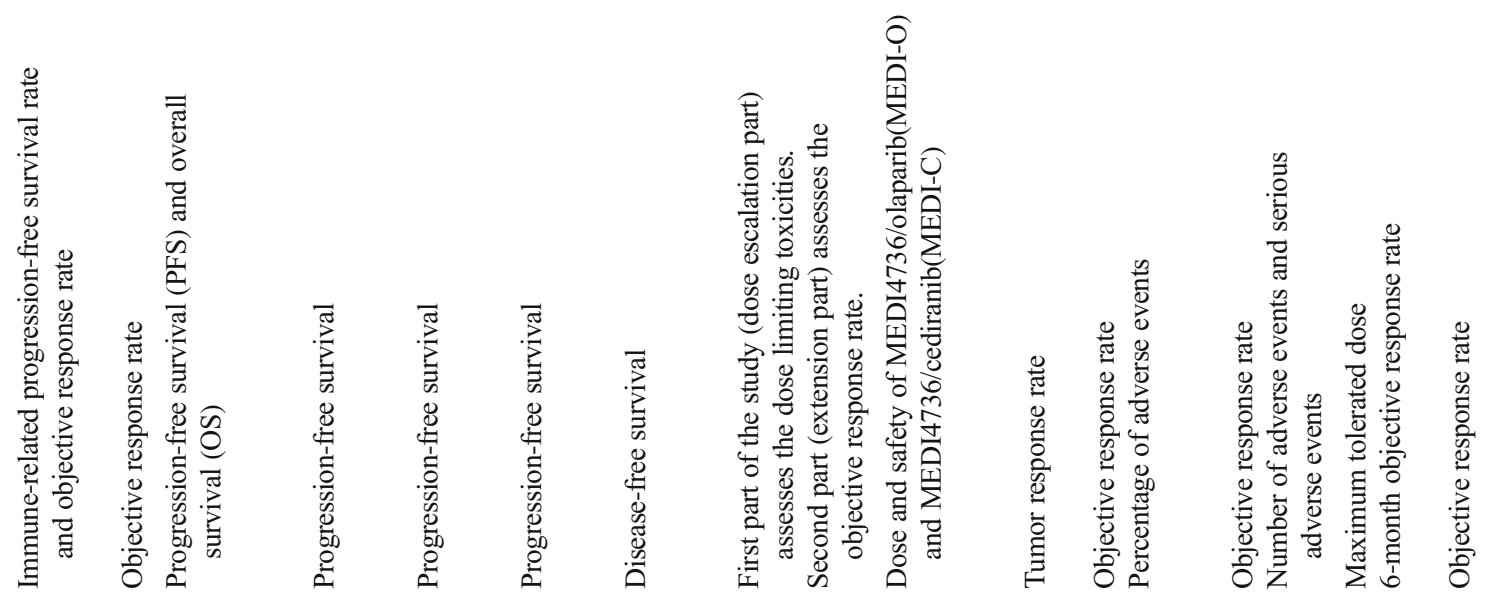

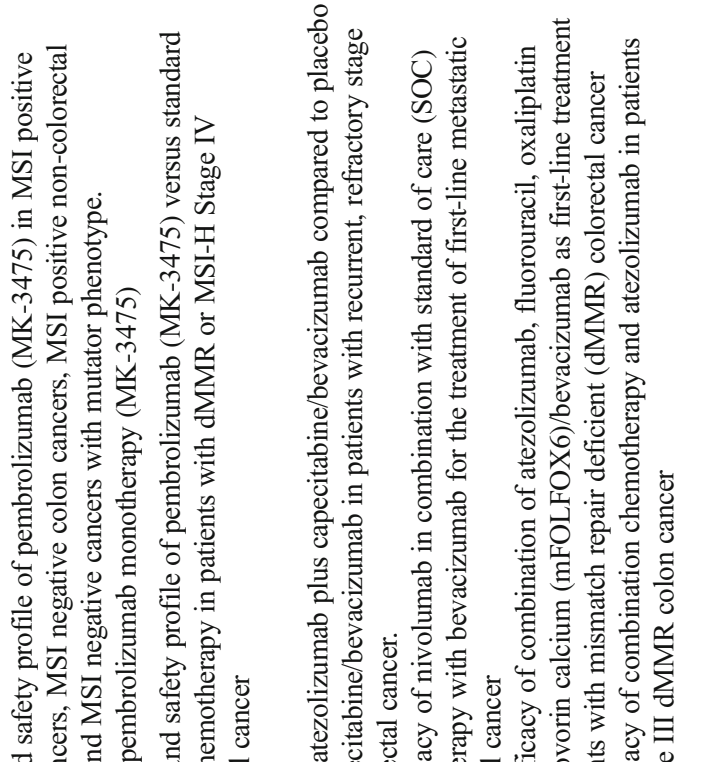

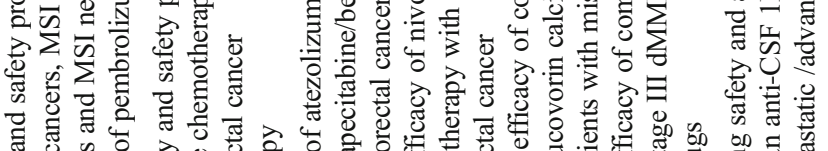

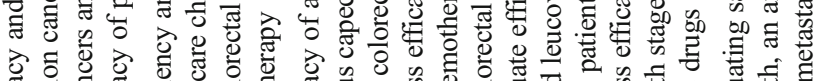

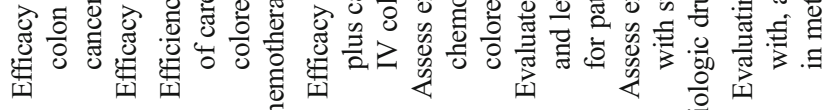
II I
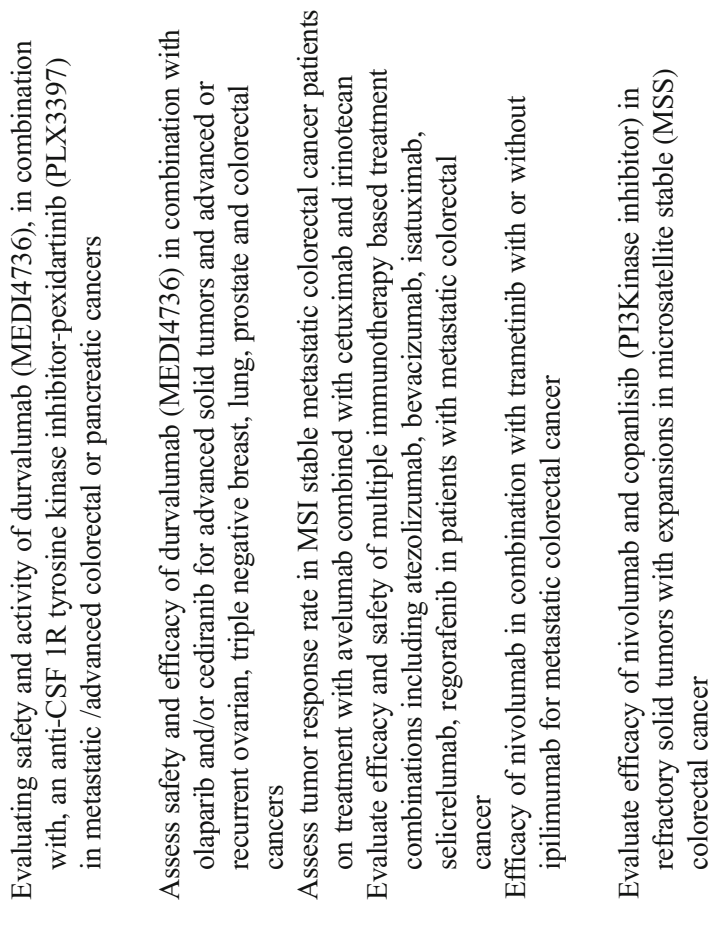

훌

है

(1)

(1)

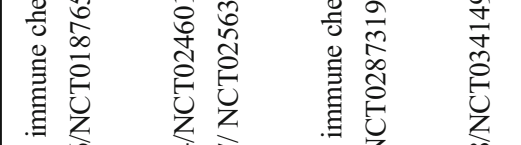

.0

를

할

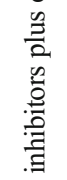

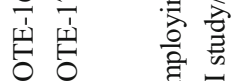

范

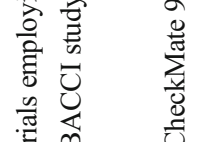

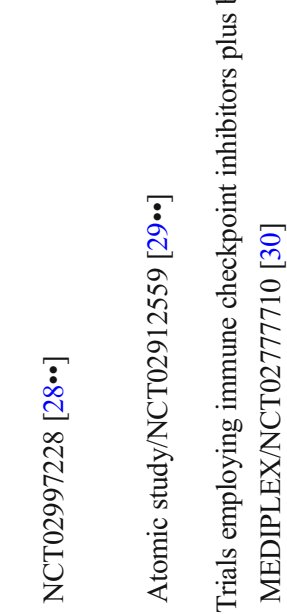

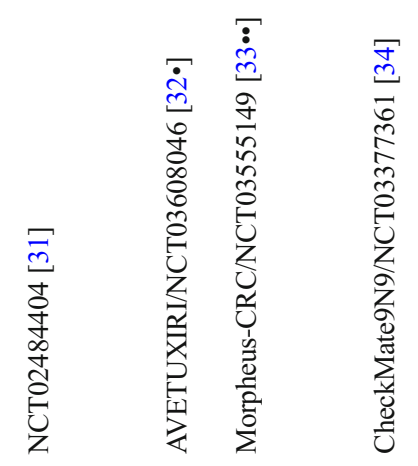

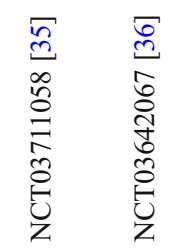




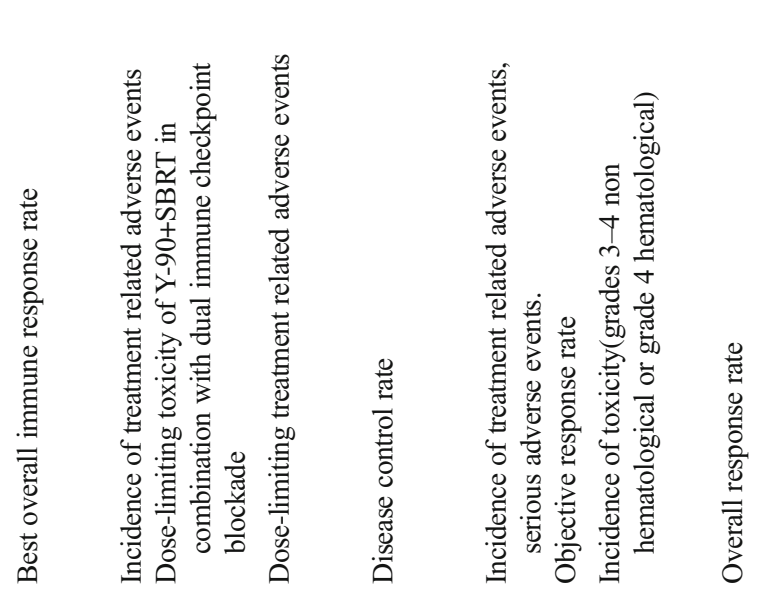

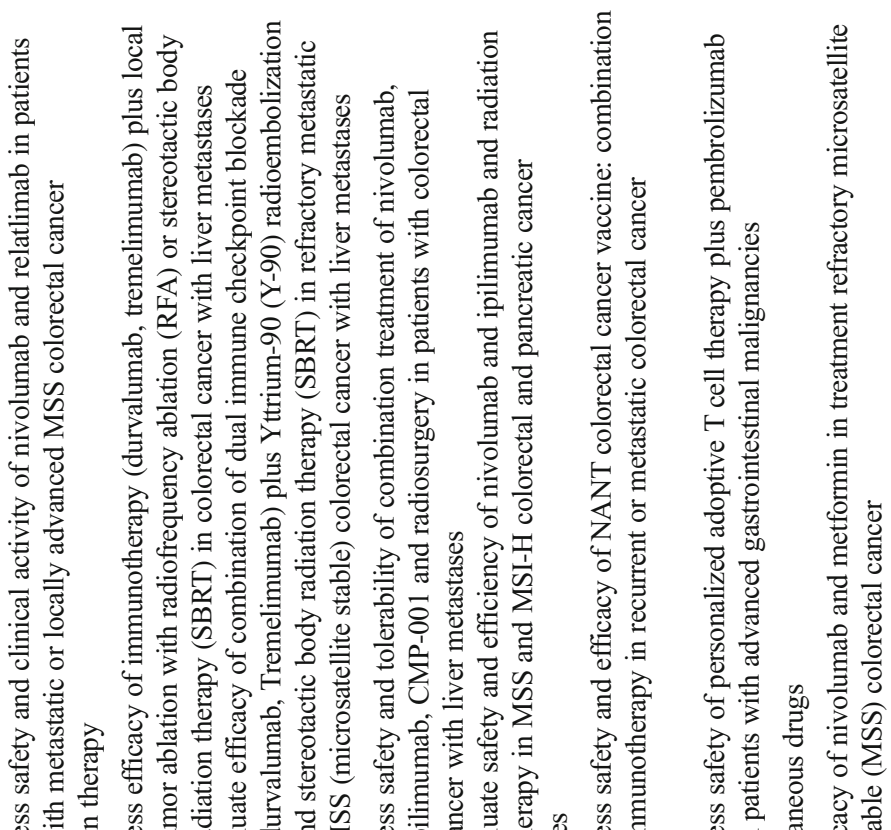

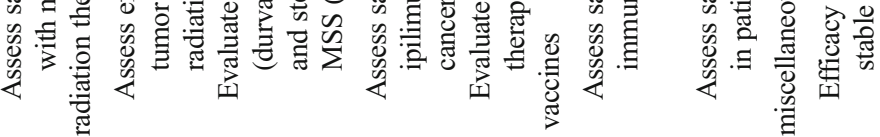

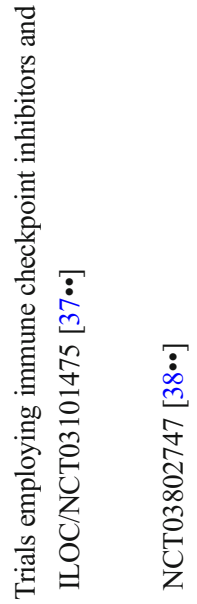

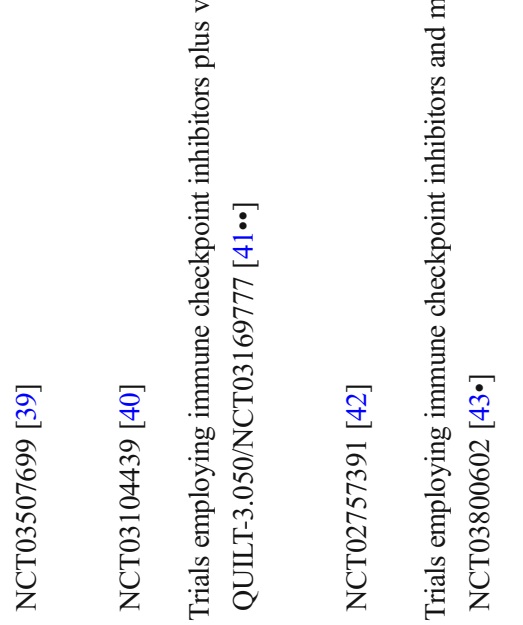


but did not eventually result in a meaningful improvement in $\mathrm{OS}$.

Other novel approaches, e.g., combination with vaccines, are also being explored as summarized in Table 3 $[44,45,46,47,48]$.

In summary, immune checkpoint inhibitors are one of the most significant advances for colorectal cancers that are $\mathrm{dMMR} / \mathrm{MSI}-\mathrm{H}$ colorectal cancers. Pembrolizumab, nivolumab, and combination therapy with nivolumab and ipilimumab have shown robust antitumor activity in this subset of patients and already are approved after failure of chemotherapy. Further developments and advances in this field will lead to patients benefiting beyond refractory setting and incorporation in earlier stages of disease. Strategies to identify how pMMR/MSS patients can benefit from immunotherapybased approaches are the most pressing questions being addressed in numerous trials.

\section{Compliance With Ethical Standards}

Conflict of Interest The authors declare that they have no conflicts of interest.

Human and Animal Rights and Informed Consent This article does not contain any studies with human or animal subjects performed by any of the authors.

Open Access This article is distributed under the terms of the Creative Commons Attribution 4.0 International License (http:// creativecommons.org/licenses/by/4.0/), which permits unrestricted use, distribution, and reproduction in any medium, provided you give appropriate credit to the original author(s) and the source, provide a link to the Creative Commons license, and indicate if changes were made.

\section{References}

Papers of particular interest, published recently, have been highlighted as:

- Of importance

-• Of major importance

1. Siegel RL, Miller KD, Jemal A. Cancer statistics, 2018. CA Cancer J Clin. 2018;68(1):7-30.

2. Koopman M, Kortman GAM, Mekenkamp L, Ligtenberg MJL, Hoogerbrugge N, Antonini NF, et al. Deficient mismatch repair system in patients with sporadic advanced colorectal cancer. Br J Cancer. 2009;100:266-73.

3. Venderbosch S, Nagtegaal ID, Maughan TS, Smith CG, Cheadle JP, Fisher D, et al. Mismatch repair status and BRAF mutation status in metastatic colorectal cancer patients: a pooled analysis of the CAIRO, CAIRO2, COIN, and FOCUS studies. Clin Cancer Res. 2014;20(20):5322-30. https://doi.org/10.1158/1078-0432.CCR14-0332.

4. Tougeron D, Cohen R, Sueur B, Sefrioui D, Gentilhomme L, Lecomte T, et al. 533PA large retrospective multicenter study evaluating prognosis and chemosensitivity of metastatic colorectal cancer with microsatellite instability. Ann Oncol. 2017;28(suppl_5):v158-208. https://doi.org/10.1093/annonc/ mdx393.

5. Nebot-Bral L, Coutzac C, Kannouche PL, Chaput N. Why is immunotherapy effective (or not) in patients with MSI/MMRD tumors? Bull Cancer. 2019;106(2):105-13. https://doi.org/10.1016/j. bulcan.2018.08.007.

6. Cohen R, Pellat A, Boussion H, Svrcek M, Lopez-Trabada D, Trouilloud I, et al. Immunotherapy and metastatic colorectal cancers with microsatellite instability or mismatch repair deficiency. Bull Cancer. 2019;106(2):137-42. https://doi.org/10.1016/j. bulcan.2018.09.004.

7. Chu JN, Choi J, Ostvar S, Torchia JA, Reynolds KL, Tramontano A, et al. Cost-effectiveness of immune checkpoint inhibitors for microsatellite instability-high/mismatch repair-deficient metastatic colorectal cancer. NCCN Guidelines Version 1.2018 Genetic/ Familial High-Risk Assessment: Colorectal. Cancer. 2019; 15;125(2):278-289. https://doi.org/10.1002/cncr.31795.

8. Llosa NJ, Cruise M, Tam A, Wicks EC, Hechenbleikner EM, Taube $\mathrm{JM}$, et al. The vigorous immune microenvironment of microsatellite instable colon cancer is balanced by multiple counter-inhibitory checkpoints. Cancer Discov. 2015;5(1):43-51.

9. NCCN Guidelines Version 1.2018 Genetic/Familial High-Risk Assessment: Colorectal. https://www.nccn.org/professionals/ physician_gls/pdf/genetics_colon.pdf.

10. Sargent DJ, Marsoni S, Monges G, Thibodeau SN, Labianca R, Hamilton SR, et al. Defective mismatch repair as a predictive marker for lack of efficacy of fluorouracil-based adjuvant therapy in colon cancer. J Clin Oncol. 2010;28(20):3219-26.

11. Le DT, et al. PD-1 blockade in tumors with mismatch-repair deficiency. N Engl J Med. 2015;372(26):2509-20.

12. O'Neil BH, et al. Safety and antitumor activity of the anti-PD-1 antibody pembrolizumab in patients with advanced colorectal carcinoma. PLoS One. 2017;12(12):e0189848.

13.• Diaz LA, et al. Pembrolizumab therapy for microsatellite instability high (MSI-H) colorectal cancer (CRC) and non-CRC. J Clin Oncol. 2017;35(15_suppl):3071-1. The early results of the KEYNOTE164 study affirmed the robust antitumor activity of pembrolizumab in patients with pretreated MSI-H colorectal cancer.

14. Overman MJ, et al. Nivolumab \pm ipilimumab in treatment (tx) of patients (pts) with metastatic colorectal cancer (mCRC) with and without high microsatellite instability (MSI-H): CheckMate-142 interim results. J Clin Oncol. 2016;34(15_suppl):3501-1.

15.• Overman MJ, et al. Durable clinical benefit with nivolumab plus ipilimumab in DNA mismatch repair-deficient/microsatellite instability-high metastatic colorectal cancer. J Clin Oncol. 2018;36(8):773-9. CheckMate-142 is the largest single-study report of combination immunotherpy with nivolumab and ipilimumab demonstrating high response rate and safety profile.

16.• Lenz HJ, van Cutsem E, Limon ML, Wong KY, Hendlisz A, Aglietta M, et al. LBA18 PR durable clinical benefit with nivolumab (NIVO) plus low-dose ipilimumab (IPI) as first-line therapy in microsatellite instability-high/mismatch repair deficient (MSI-H/dMMR) metastatic colorectal cancer (mCRC). Ann Oncol. 2018;29(Suppl 8). This study reports the first results of clinical efficacy and safety of nivolumab plus a low dose of ipilimumab as a new first-line therapy for dMMR/MSI-H mCRC.

17.• Chalabi M, Fanchi LF, Van den Berg JG, Beets GL, Lopez-Yurda M, Aalbers AG, et al. LBA37_PRNeoadjuvant ipilimumab plus nivolumab in early stage colon cancer. Ann Oncol. 2018;29(suppl_8). https://doi.org/10.1093/annonc/mdy424.047. The first study to suggest short-term neoadjuvant nivolumab plus ipilimumab combination therapy has the potential to 
change current standard of care in dMMR early satge colon cancers.

18. NCCN Guidelines Version 2.2019 Colon Cancer. Systemic therapy for advanced or metastatic disease - chemotherapy regimens. https://www.nccn.org/professionals/physician gls/pdf/colon.pdf.

19. Bendell J, Ciardiello F, Tabernero J, Tebbutt N, Eng C, Di Bartolomeo M, et al. LBA-004 efficacy and safety results from IMblaze 370, a randomised phase III study comparing atezolizumab+cobimetinib and atezolizumab monotherapy vs regorafenib in chemotherapy-refractory metastatic colorectal cancer. Ann Oncol. 2018;29(suppl 5). https://doi.org/10.1093/annonc/ mdy208.003. A global, multi-center study whose primary analysis validated that atezolizumab plus cobimetinib combination or atezolizumab monotherapy had no statistically significant outcomes in efficay for MSS mCRC.

20. Chen EX, Jonker DJ, Kennecke HF, Berry SR, Couture F, Ahmad CE, et al. 26 trial: a phase II randomized study of durvalumab (D) plus tremelimumab (T) and best supportive care (BSC) versus BSC alone in patients (pts) with advanced refractory colorectal carcinoma (rCRC). J Clin Oncol. 2019;37(4_suppl):481-1. https://doi.org/ 10.1200/JCO.2019.37.4.

21. Formenti SC, Demaria S. Combining radiotherapy and cancer immunotherapy: a paradigm shift. J Natl Cancer Inst. 2013;105(4): 256-65.

22. Reits EA, Hodge JW, Herberts CA, Groothuis TA, Chakraborty M, K.Wansley E, et al. Radiation modulates the peptide repertoire, enhances MHC class I expression, and induces successful antitumor immunotherapy. J Exp Med. 2006;203(5):1259-71.

23. NIH U.S. National Library of Medicine ClinicalTrials.gov. Phase 2 Study of MK-3475 in Patients With Microsatellite Unstable (MSI) Tumors. ClinicalTrials.gov Identifier: NCT01876511. https:// clinicaltrials.gov/ct2/show/NCT01876511.

24. NIH U.S. National Library of Medicine ClinicalTrials.gov. Study of Pembrolizumab (MK-3475) as Monotherapy in Participants With Previously-Treated Locally Advanced Unresectable or Metastatic Colorectal Cancer (MK-3475-164/KEYNOTE-164) ClinicalTrials.gov Identifier: NCT02460198. https://clinicaltrials. gov/ct2/show/NCT02460198.

25. NIH U.S. National Library of Medicine ClinicalTrials.gov. Study of Pembrolizumab (MK-3475) vs Standard Therapy in Participants With Microsatellite Instability-High (MSI-H) or Mismatch Repair Deficient (dMMR) Stage IV Colorectal Carcinoma (MK-3475-177/ KEYNOTE-177) ClinicalTrials.gov Identifier: NCT02563002. https://clinicaltrials.gov/ct2/show/NCT02563002. An ongoing clinical trial that compares progression free survival and overall survival in dMMR/MSI-H stage IV CRC patients receiving pembrolizumab vs those receiving standard chemotherapy.

26. NIH U.S. National Library of Medicine ClinicalTrials.gov. Capecitabine and Bevacizumab With or Without Atezolizumab in Treating Patients With Refractory Metastatic Colorectal Cancer ClinicalTrials.gov Identifier: NCT02873195. https://clinicaltrials. gov/ct2/show/NCT02873195. BACCI study is an ongoing clinical trial to estimate the efficacy of atezolizumab plus capecitabine/bevacizumab therapy in refractory $\mathrm{mCRC}$ patients.

27. NIH U.S. National Library of Medicine ClinicalTrials.gov. An Investigational Immunotherapy Study of Nivolumab With Standard of Care Therapy vs Standard of Care Therapy for FirstLine Treatment of Colorectal Cancer That Has Spread (CheckMate 9X8) ClinicalTrials.gov Identifier: NCT03414983. https:// clinicaltrials.gov/ct2/show/NCT03414983.

28.• NIH U.S. National Library of Medicine ClinicalTrials.gov. Combination Chemotherapy, Bevacizumab, and/or Atezolizumab in Treating Patients With Deficient DNA Mismatch Repair Metastatic Colorectal Cancer ClinicalTrials.gov Identifier:
NCT02997228. https:/clinicaltrials.gov/ct2/show/NCT02997228. This phase III randomized clinical trial is currently recruiting patients to study the efficacy of atezolizumab plus combination chemotherapy/bevacizumab as first line tresatment of patients with dMMR mCRC.

29.• NIH U.S. National Library of Medicine ClinicalTrials.gov. Combination Chemotherapy With or Without Atezolizumab in Treating Patients With Stage III Colon Cancer and Deficient DNA Mismatch Repair ClinicalTrials.gov Identifier: NCT02912559. https://clinicaltrials.gov/ct2/show/NCT02912559. Atomic study is an ongoing phase III clinical trial to assess efficacy of combination chemotherapy and atezolizumab in patients with stage III dMMR colon cancer.

30. NIH U.S. National Library of Medicine ClinicalTrials.gov. Evaluation of Safety and Activity of an Anti-PDL1 Antibody (DURVALUMAB) Combined With CSF-1R TKI (PEXIDARTINIB) in Patients With Metastatic/Advanced Pancreatic or Colorectal Cancers (MEDIPLEX) ClinicalTrials.gov Identifier: NCT02777710. https://clinicaltrials.gov/ct2/show/ NCT02777710.

31. NIH U.S. National Library of Medicine ClinicalTrials.gov. Phase I/ II Study of the Anti-Programmed Death Ligand-1 Antibody MEDI4736 in Combination With Olaparib and/or Cediranib for Advanced Solid Tumors and Advanced or Recurrent Ovarian, Triple Negative Breast, Lung, Prostate and Colorectal Cancers. ClinicalTrials.gov Identifier: NCT02484404. https://clinicaltrials. gov/ct2/show/NCT02484404.

32. NIH U.S. National Library of Medicine ClinicalTrials.gov. Avelumab Combined With Cetuximab and Irinotecan for Treatment Refractory Metastatic Colorectal Microsatellite Stable Cancer (AVETUXIRI). ClinicalTrials.gov Identifier: NCT03608046. https://clinicaltrials.gov/ct2/show/NCT03608046. A new clinical trial to assess tumor response rate in MSI stable metastatic colorectal cancer patients on treatment with avelumab combined with cetuximab and irinotecan.

33.• NIH U.S. National Library of Medicine ClinicalTrials.gov. A Study Evaluating the Efficacy and Safety of Multiple ImmunotherapyBased Treatment Combinations in Patients With Metastatic Colorectal Cancer (Morpheus-CRC). ClinicalTrials.gov Identifier: NCT03555149. https://clinicaltrials.gov/ct2/show/NCT03555149. Morpheus-CRC is a phase Ib/II, open-label, multicenter, randomized study designed to evaluate safety and efficacay of multiple immunotherapy based therapy combinations.

34. NIH U.S. National Library of Medicine ClinicalTrials.gov. An Investigational Immuno-therapy Study Of Nivolumab In Combination With Trametinib With Or Without Ipilimumab In Patients With Previously Treated Cancer of the Colon or Rectum That Has Spread (CheckMate 9N9). ClinicalTrials.gov Identifier: NCT03377361. https://clinicaltrials.gov/ct2/show/NCT03377361.

35. NIH U.S. National Library of Medicine ClinicalTrials.gov. Study of PI3Kinase Inhibition (Copanlisib) and Anti-PD-1 Antibody Nivolumab in Relapsed/Refractory Solid Tumors With Expansions in Mismatch-repair Proficient (MSS) Colorectal Cancer ClinicalTrials.gov Identifier: NCT03711058. https:// clinicaltrials.gov/ct2/show/NCT03711058.

36. NIH U.S. National Library of Medicine ClinicalTrials.gov. Study of Nivolumab and Relatlimab in Patients With Microsatellite Stable (MSS) Advanced Colorectal Cancer ClinicalTrials.gov Identifier: NCT03642067. https://clinicaltrials.gov/ct2/show/NCT03642067.

$37 . \bullet$ NIH U.S. National Library of Medicine ClinicalTrials.gov. Synergism of Immunomodulation and Tumor Ablation (ILOC) ClinicalTrials.gov Identifier: NCT03101475. https://clinicaltrials. gov/ct2/show/NCT03101475. ILOC- a phase II single arm, multicenter trial to study the synergism of immunotherapy (durvalumab, tremelimumab) and local tumor ablation with 
radiofrequency ablation (RFA) or stereotactic body radiation therapy (SBRT) in colorectal cancer with liver metastases.

38.• NIH U.S. National Library of Medicine ClinicalTrials.gov. Immunotherapy Combined With Y-90 and SBRT for Colorectal Liver Metastases ClinicalTrials.gov Identifier: NCT03802747. https://clinicaltrials.gov/ct2/show/NCT03802747. A new phse I clinical trial to evaluate the efficacy of combination of dual immune checkpoint blockade (durvalumab, Tremelimumab) plus Yttrium-90 (Y-90) radioembolization \& stereotactic body radiation therapy (SBRT) in refractory metastatic MSS colorectal cancer with liver metastases.

39. NIH U.S. National Library of Medicine ClinicalTrials.gov. Combined Immunotherapy and Radiosurgery for Metastatic Colorectal Cancer ClinicalTrials.gov Identifier: NCT03507699. https://clinicaltrials.gov/ct2/show/NCT03507699.

40. NIH U.S. National Library of Medicine ClinicalTrials.gov. Nivolumab and Ipilimumab and Radiation Therapy in MSS and MSI High Colorectal and Pancreatic Cancer ClinicalTrials.gov Identifier: NCT03104439. https://clinicaltrials.gov/ct2/show/ NCT03104439.

41.• NIH U.S. National Library of Medicine ClinicalTrials.gov. QUILT3.050: NANT Colorectal Cancer (CRC) Vaccine: Combination Immunotherapy in Subjects With Recurrent or Metastatic CRC ClinicalTrials.gov Identifier: NCT03169777. https://clinicaltrials. gov/ct2/show/NCT03169777. QUILT-3.050 - phase 1b/2 study to assess the safety and efficacy of NANT colorectal cancer vaccine: combination immunotherapy in recurrent or metastatic colorectal cancer.

42. NIH U.S. National Library of Medicine ClinicalTrials.gov. CD8+ T Cell Therapy and Pembrolizumab in Treating Patients With Metastatic Gastrointestinal Tumors ClinicalTrials.gov Identifier: NCT02757391. https://clinicaltrials.gov/ct2/show/NCT02757391.
43. NIH U.S. National Library of Medicine ClinicalTrials.gov. Nivolumab and Metformin in Patients With Treatment Refractory MSS Colorectal Cancer ClinicalTrials.gov Identifier: NCT03800602. https:/clinicaltrials.gov/ct2/show/NCT03800602. A phse II trial to determine the efficacy of nivolumab and metformin in treatment refractory microsatellite stable (MSS) colorectal cancer.

44. Fletcher R, Wang YJ, Schoen RE, Finn OJ, Yu J, Zhang L. Colorectal cancer prevention: immune modulation taking the stage. Biochim Biophys Acta Rev Cancer. 2018;1869(2):138-48.

45. Kimura T, McKolanis JR, Dzubinski LA, Islam K, Potter DM, Salazar AM, et al. MUC1 vaccine for individuals with advanced adenoma of the colon: a cancer immunoprevention feasibility study. Cancer Prev Res (Phila). 2013;6(1):18-26.

46. Morse MA, Chaudhry A, Gabitzsch ES, Hobeika AC, Osada T, Clay TM, et al. Novel adenoviral vector induces T-cell responses despite anti-adenoviral neutralizing antibodies in colorectal cancer patients. Cancer Immunol Immunother. 2013;62(8):1293-301.

47. Koblansky AA, Truax AD, Liu R, Montgomery SA, Ding S, Wilson JE, et al. The innate immune receptor NLRX1 functions as a tumor suppressor by reducing colon tumorigenesis and key tumor-promoting signals. Cell Rep. 2016;14(11):2562-75.

48. Zelenay S, van der Veen AG, Böttcher JP, Snelgrove KJ, Rogers N, Acton SE, et al. Cyclooxygenase-dependent tumor growth through evasion of immunity. Cell. 2015;162(6):1257-70.

Publisher's Note Springer Nature remains neutral with regard to jurisdictional claims in published maps and institutional affiliations. 